\title{
ON THE STABILITY OF CONVEX SUMS OF RANK-1 PERTURBED MATRICES
}

By

\author{
THOMAS LAFFEY \\ Department of Mathematics, University College Dublin \\ ROBERT SHORTEN \\ Hamilton Institute, National University of Ireland, Maynooth
}

and

\author{
Fiacre Ó CAIrbre*
}

Department of Mathematics, National University of Ireland, Maynooth

[Received 14 November 2002. Read 16 March 2004. Published November 2004.]

\section{Abstract}

New compact conditions are obtained that guarantee stability of a convex sum of a pair of matrices. It is conjectured that these conditions are both necessary and sufficient for the stability of a pair of related convex sums. It is anticipated that the proof presented for a single pencil will be modified to yield conditions for both pencils simultaneously.

\section{Introduction}

We consider the problem of determining appropriate conditions on the matrices $A_{1}$ and $A_{2}$ that are necessary and sufficient for the locus of eigenvalues of the convex sums (pencils)

$$
\begin{array}{cc}
\alpha A_{1}+(1-\alpha) A_{2}, & \alpha \in[0,1], \\
\alpha A_{1}^{-1}+(1-\alpha) A_{2}, & \alpha \in[0,1],
\end{array}
$$

to remain in $\mathbb{C}^{-}$(the open left half of the complex plane) for all $\alpha \in[0,1]$, where $A_{1}$, $A_{2}$ are real $n \times n$ matrices and $A_{2}=A_{1}+B$, where rank $B=1$. Problems of this nature can be readily found in the literature on robust stability of linear systems [1]. More recently research on the stability of switching systems constructed by switching between two linear systems [2], has illustrated the importance of convex sums of the form of Equations (1.1) and (1.2): under certain situations the stability of the pair of pencils is necessary and sufficient for the existence of a common quadratic Lyapunov function for the dynamic systems $\dot{x}=A_{i} x, i \in\{1,2\}$, where $A_{1}$ and $A_{2}$ are stable matrices. In this paper we begin the study of establishing compact conditions that are necessary and sufficient for the stability of both convex sums simultaneously by

*Corresponding author; e-mail: foc@maths.may.ie 
presenting sufficient conditions for the stability of a single convex sum. While problems of this nature have been widely discussed in the literature, and may be considered solved by some authors [1;3], the results presented in this paper are important for a number of reasons. First, in contrast to other results in the literature (which are expressed using higher dimensional matrices like Kronecker sums), our results are expressed in terms of the original matrices $A_{1}, A_{2}$. Second, our results illustrate the remarkable connection between the convex sum (1.1) and the convex sum (1.2); namely that instability of the convex sum (1.1) by eigenvalues passing through the imaginary axis (excluding the origin) immediately implies the instability of the convex sum (1.2) by eigenvalues passing through the origin of the complex plane. This observation is likely to have profound consequences for the stability of switching systems. Finally, the proof of our conditions is based on the original matrices $A_{1}$ and $A_{2}$, and hence differs considerably from treatments of similar problems in the literature $[1 ; 3]$.

This paper is structured as follows: Section 2 presents the main result of the paper and Section 3 contains examples to illustrate our result. In Section 4 we briefly discuss our conclusions.

\section{The main result}

We begin our discussion with the following definition:

Definition. A real $n \times n$ matrix is defined to be stable if all its eigenvalues have negative real part.

We prove the following Lemma before we prove the main result of this paper.

Lemma 2.1. Suppose $A_{1}$ and $A_{2}=A_{1}+B$ are real $n \times n$ stable matrices and that $B$ has rank one. Then $A_{1}^{-1} A_{2}$ has no negative eigenvalues.

Proof. $A_{1}^{-1} A_{2}=I+A_{1}^{-1} B$, where $A_{1}^{-1} B$ has rank one. Thus, $A_{1}^{-1} B$ has at most one non-zero eigenvalue and that eigenvalue (call it $\lambda$ if it exists) has algebraic multiplicity one. Note that $1+\lambda \geq 0$ since otherwise $\operatorname{det} A_{1}^{-1} A_{2}<0$, which is impossible because $A_{1}^{-1}$ and $A_{2}$ are both stable and so their determinants have the same sign. Thus, the eigenvalues of $A_{1}^{-1} A_{2}$, which are either 1 or $1+\lambda$ (if $\lambda$ exists), are all nonnegative.

The main result of this paper is the following theorem, which gives sufficient conditions for the stability of a convex sum of two matrices.

Theorem 2.2. Suppose $A_{1}$ and $A_{2}=A_{1}+B$ are real $n \times n$ stable matrices and that $B$ has rank one. Suppose that $A_{1} A_{2}$ has no negative eigenvalues. Then $\alpha A_{1}+(1-\alpha) A_{2}$ is stable for all $\alpha$ with $0 \leq \alpha \leq 1$. 
ProOF. We will prove the result by contradiction. Suppose that for some $\alpha$ with $0<\alpha<1$, we have that $\alpha A_{1}+(1-\alpha) A_{2}$ is unstable. Then there exists an $\alpha_{0}$ with $0<\alpha_{0}<1$ such that $\alpha_{0} A_{1}+\left(1-\alpha_{0}\right) A_{2}$ is unstable and has a purely imaginary eigenvalue $i \gamma$ (with $\gamma$ real). Note that $\alpha_{0} A_{1}+\left(1-\alpha_{0}\right) A_{2}=A_{1}+\left(1-\alpha_{0}\right) B$. Let $\delta=1-\alpha_{0}$. Thus, $A_{1}+\delta B$ is unstable and has a purely imaginary eigenvalue $i \gamma$ ( $\gamma$ real) for some $\delta$ with $0<\delta<1$.

Note that $\gamma \neq 0$ since otherwise $\operatorname{det}\left(\alpha_{0} A_{1}+\left(1-\alpha_{0}\right) A_{2}\right)=0$ (for some $\alpha_{0}$ with $\left.0<\alpha_{0}<1\right)$, which implies that $\operatorname{det}\left(\alpha_{0} I+\left(1-\alpha_{0}\right) A_{1}^{-1} A_{2}\right)=0$ and so $A_{1}^{-1} A_{2}$ has a negative eigenvalue $\frac{\alpha_{0}}{\alpha_{0}-1}$, which is false. Thus, $i \gamma$ and $-i \gamma$ are distinct eigenvalues of $A_{1}+\delta B$. Let $v_{1}, v_{2}$ be eigenvectors corresponding to $i \gamma,-i \gamma$, respectively. We may assume that $v_{1}=u_{1}+i u_{2}$ and $v_{2}=u_{1}-i u_{2}$, where $u_{1}, u_{2}$ are real vectors. We get that $u_{1}$ and $u_{2}$ are linearly independent over the reals. Let $U$ be the real subspace spanned by $u_{1}$ and $u_{2}$. Note that

$$
\left(\left(A_{1}+\delta B\right)^{2}+\gamma^{2} I\right) u=0, \quad \forall u \in U .
$$

Let $K=\left\{w \in \mathbb{R}^{n}: B w=0\right\}$ be the kernel of $B$. We have $\operatorname{dim} K=n-1$ since $B$ has rank one. Also, $\operatorname{dim} U=2$ and so

$$
\operatorname{dim}(K \cap U) \geq 1
$$

because $n \geq \operatorname{dim}(K+U)=\operatorname{dim} K+\operatorname{dim} U-\operatorname{dim}(K \cap U)=n+1-\operatorname{dim}(K \cap U)$.

Let $u$ be a non-zero element in $K \cap U$. Then $B u=0$ and so (1) implies that

$$
\left(\left(A_{1}+\delta B\right) A_{1}+\gamma^{2} I\right) u=0 .
$$

Thus, $\left(A_{1}+\delta B\right) A_{1}$ has $-\gamma^{2}$ as an eigenvalue. Hence, $A_{1}\left(A_{1}+\delta B\right)$ has $-\gamma^{2}$ as an eigenvalue, and we obtain

$$
\operatorname{det}\left(A_{1}\left(A_{1}+\delta B\right)+\gamma^{2} I\right)=0 .
$$

For $x \geq 0$ and $y>0$ we now define

$$
f(x, y)=\operatorname{det}\left(A_{1}\left(A_{1}+y B\right)+x I\right) .
$$

Note that $A_{1}^{2}$ (and hence $A_{1}^{-2}$ ) has no eigenvalues less than or equal to zero since $A_{1}$ is stable. Thus, $I+x A_{1}^{-2}$ is invertible for $x \geq 0$. Also,

$$
f(x, y)=\left(\operatorname{det} A_{1}\right)^{2} \operatorname{det}\left(I+x A_{1}^{-2}\right) g(x, y),
$$

where

$$
g(x, y)=\operatorname{det}\left(I+y A_{1}^{-1} B\left(I+x A_{1}^{-2}\right)^{-1}\right) .
$$

Note that $C=A_{1}^{-1} B\left(I+x A_{1}^{-2}\right)^{-1}$ has rank one and therefore it has at most one non-zero eigenvalue and that eigenvalue (if it exists) has algebraic multiplicity one. Thus, $\operatorname{tr} C$ is either the unique non-zero eigenvalue or else it is zero, where $\operatorname{tr}$ denotes the trace of a matrix. Consequently,

$$
g(x, y)=1+y \operatorname{tr}\left(A_{1}^{-1} B\left(I+x A_{1}^{-2}\right)^{-1}\right) .
$$

We define 


$$
h(x)=\operatorname{tr}\left(A_{1}^{-1} B\left(I+x A_{1}^{-2}\right)^{-1}\right)
$$

so that $g(x, y)=1+y h(x)$. Note that $g\left(\gamma^{2}, \delta\right)=0$ and so $h\left(\gamma^{2}\right)=-\frac{1}{\delta}<-1$.

Now $h(0)=\operatorname{tr}\left(A_{1}^{-1} B\right)$. Note that $A_{1}^{-1} A_{2}=I+A_{1}^{-1} B$, where $A_{1}^{-1} B$ has rank one. Thus, $A_{1}^{-1} B$ has at most one non-zero eigenvalue and that eigenvalue (if it exists) has algebraic multipicity one. So $\operatorname{tr}\left(A_{1}^{-1} B\right)$ is either the unique non-zero eigenvalue or else it is zero. Consequently,

$$
\operatorname{tr}\left(A_{1}^{-1} B\right) \geq-1
$$

because the eigenvalues of $I+A_{1}^{-1} B$ (which are of the form 1 or $1+\operatorname{tr}\left(A_{1}^{-1} B\right)$ ) cannot be negative. Thus, $h(0) \geq-1$. In fact $h(0)>-1$ since otherwise $\operatorname{tr}\left(A_{1}^{-1} B\right)=$ -1 , and so $\operatorname{det}\left(I+A_{1}^{-1} B\right)=0$, which implies that $\operatorname{det}\left(A_{1}+B\right)=0$, which is false.

Now $h$ is a continuous function for $x \geq 0$, and so by the Intermediate Value Theorem we have that there exists a $z$ with $0<z<\gamma^{2}$ such that $h(z)=-1$. Thus, $g(z, 1)=0$ and so $f(z, 1)=0$. Therefore

$$
\operatorname{det}\left(A_{1}\left(A_{1}+B\right)+z I\right)=0
$$

and so $A_{1} A_{2}=A_{1}\left(A_{1}+B\right)$ has $-z$ as an eigenvalue, which is a contradiction.

Note. See Section 3 for a numerical example where $A_{1}$ and $A_{2}$ are stable matrices with a rank one difference and $A_{1} A_{2}$ has a negative eigenvalue, but the convex sum $\alpha A_{1}+$ $(1-\alpha) A_{2}$ is still stable $\forall \alpha \in[0,1]$.

The following corollary shows a surprising connection between the convex sums (1.1) and (1.2).

Corollary 2.3. Suppose $A_{1}$ and $A_{2}=A_{1}+B$ are real $n \times n$ stable matrices and that $B$ has rank one. Suppose that $\alpha A_{1}+(1-\alpha) A_{2}$ is invertible $\forall \alpha \in[0,1]$ and that there exists a $\beta$ with $0<\beta<1$ such that $\beta A_{1}+(1-\beta) A_{2}$ has a non-zero purely imaginary eigenvalue, i.e. the convex sum $\alpha A_{1}+(1-\alpha) A_{2}$ is unstable by eigenvalues passing through the imaginary axis (excluding the origin). Then there exists a with $0<\tau<1$ such that $\tau A_{1}^{-1}+(1-\tau) A_{2}$ has a zero eigenvalue, i.e. the convex sum $\alpha A_{1}^{-1}+(1-$ $\alpha) A_{2}$ is unstable with an eigenvalue passing through the origin in the complex plane.

Proof. Theorem 2 implies that $A_{1} A_{2}$ has a negative eigenvalue $z$. Thus $\operatorname{det}\left(A_{1} A_{2}-\right.$ $z I)=0$. Thus

$$
\operatorname{det}\left(A_{2}-z A_{1}^{-1}\right)=0
$$

This means that

$$
\operatorname{det}\left(\frac{1}{1-z} A_{2}-\frac{z}{1-z} A_{1}^{-1}\right)=0 .
$$

Hence, there exists a $\tau$ with $0<\tau<1$ such that $\tau A_{1}^{-1}+(1-\tau) A_{2}$ has a zero eigenvalue. 


\section{Numerical examples}

In this section we present numerical examples that illustrate the main features of our result and its proof.

Example 1. The conditions of Theorem 2.2 are not necessary.

The conditions of the theorem are only sufficient for the stability of the convex sum $\alpha A_{1}+(1-\alpha) A_{2}$. This fact is clearly illustrated with the following matrices.

$$
\begin{aligned}
& A_{1} \approx\left[\begin{array}{rrrr}
0.289 & 0.323 & 0.397 & -0.109 \\
-0.477 & -0.283 & -0.175 & -0.294 \\
-0.139 & 0.252 & -0.465 & 0.377 \\
-0.099 & 0.060 & -0.047 & -0.581
\end{array}\right], \\
& A_{2} \approx\left[\begin{array}{rrrr}
0.253 & 0.336 & 0.479 & -0.142 \\
-0.481 & -0.281 & -0.166 & -0.298 \\
-0.126 & 0.247 & -0.495 & 0.389 \\
-0.055 & 0.044 & -0.147 & -0.540
\end{array}\right] .
\end{aligned}
$$

The eigenvalues of the matrix product $A_{1} A_{2}$ are $\{0.223+0.191 i, 0.223-$ $0.191 i,-0.187,-0.166\}$ and so $A_{1} A_{2}$ has a negative eigenvalue. However, the convex sum is stable, as depicted by the evolution of the locus of eigenvalues of $\alpha A_{1}+(1-$

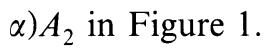

Example 2. Main feature of the proof of Theorem 2.2.

The main idea in the proof is that $A_{1} A_{2}$ must have a negative eigenvalue if the pencil $\alpha A_{1}+(1-\alpha) A_{2}$ has a purely imaginary eigenvalue. This is illustrated with the following matrices. Let

$$
A_{1} \approx\left[\begin{array}{lrrr}
-0.740 & -6.975 & -0.514 & 1.683 \\
-0.045 & 0.514 & -4.686 & 2.518 \\
-0.0530 & -2.813 & -2.295 & 6.308 \\
-0.0527 & 3.2367 & -6.792 & 0.442
\end{array}\right]
$$

and let

$$
A_{2} \approx\left[\begin{array}{lllr}
-0.740 & -6.990 & -0.508 & 1.689 \\
-0.0408 & -3.0941 & -3.3633 & 3.903 \\
-0.051 & -3.979 & -1.868 & 6.755 \\
-0.059 & 8.214 & -8.617 & -1.466
\end{array}\right]
$$

The matrices $A_{1}, A_{2}$ are stable and satisfy the conditions of the theorem except the negative eigenvalue condition. The evolution of the locus of eigenvalues of $\alpha A_{1}+$ $(1-\alpha) A_{2}$ is depicted in Figure 2. As can be seen, the pencil fails to be stable for some $\alpha \in[0,1]$ and becomes unstable through the imaginary axis. According to our main result this implies that some eigenvalues of $A_{1} A_{2}$ are negative; the eigenvalues of $A_{1} A_{2}$ are $\{-25.306,0.603,-16.130,5.085\}$. 


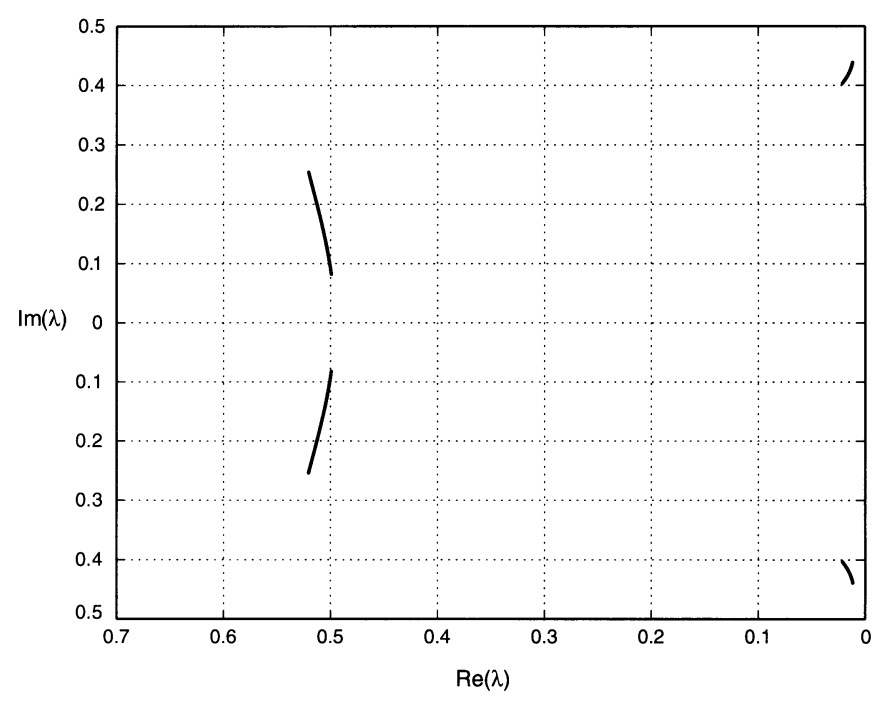

FIG. 1 - Non-necessity of conditions.

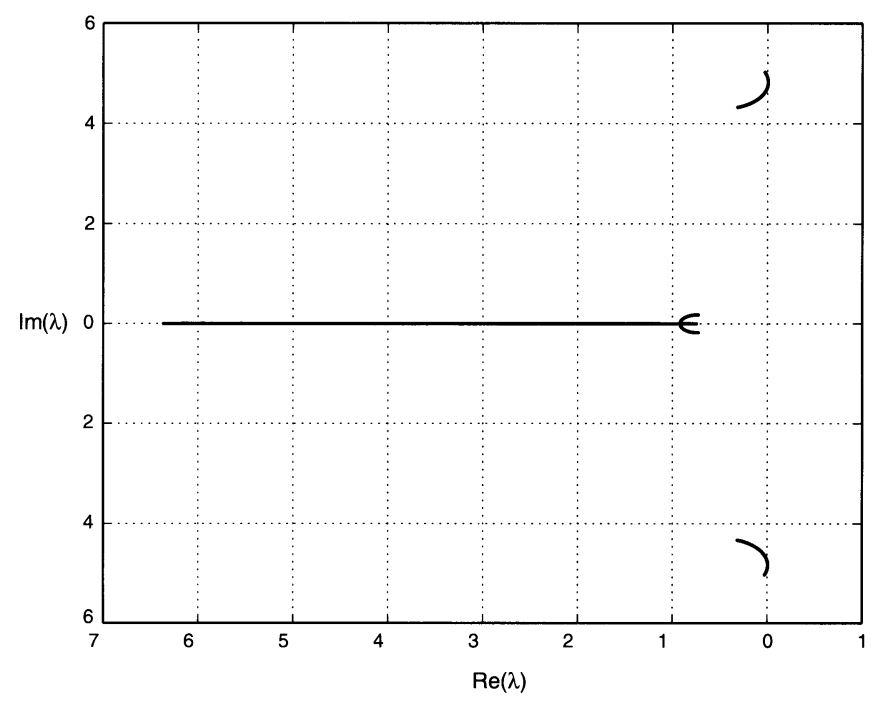

FIG. 2-Illustration of Example 2.

\section{Conclusions}

The main result of this paper gives sufficient conditions for when the convex sum of two matrices is stable. We also prove a surprising connection between the two convex sums (1.1) and (1.2). This paper begins the study of establishing compact necessary and sufficient conditions for the simultaneous stability of the two convex sums (1.1) 
and (1.2). We hope to employ the technique in the proof of Theorem 2.2 to prove the following conjecture:

Conjecture. Suppose $A_{1}$ and $A_{2}=A_{1}+B$ are real $n \times n$ stable matrices and that $B$ has rank one. Then $A_{1} A_{2}$ has no negative eigenvalues if and only if both $\alpha A_{1}+(1-\alpha) A_{2}$ and $\alpha A_{1}^{-1}+(1-\alpha) A_{2}$ are stable for all $\alpha$ with $0 \leq \alpha \leq 1$.

\section{ACKNOWLEDGEMENTS}

This work was partially supported by the European Union funded research training network Multi-Agent Control, HPRN-CT-1999-00107 ${ }^{1}$ and by the Enterprise Ireland grant SC/2000/084/Y.

\section{REFERENCES}

[1] M. Fu and B. Barmish. Stability of convex and linear combinations of polynomials and matrices arising in robustness problems. Proceedings of the Conference on Information Sciences and Systems (1987), 16-21. John Hopkins University.

[2] R. Shorten and K.S. Narendra. On the existence of a common Lyapunov function for linear stable switching systems. Proceedings of the 10th Yale workshop on adaptive and learning systems (1998), 130-40.

[3] S. Bialas. A necessary and sufficient condition for the stability of convex combinations of stable polynomials or matrices. Bulletin of the Polish Academy of Sciences 33 (9-10) (1985), 473-80.

1 This work is the sole responsibility of the authors and does not reflect the European Union's opinion. The EU is not responsible for any use of data appearing in this publication. 